\title{
Conhecimento que acompanhantes de pacientes de uma clínica-escola de Fonoaudiologia tem sobre a atuação fonoaudiológica
}

\author{
Knowledge persons accompanying patients in a Speech- \\ Language Pathology school-clinic have about the work of \\ speech-language pathologists
}

\author{
Ana Gabriela Lopes Pimentel ${ }^{1}$, Simone Aparecida Lopes-Herrera ${ }^{2}$, Tâmyne Ferreira Duarte ${ }^{3}$
}

\begin{abstract}
RESUMO
Objetivo: Avaliar o conhecimento dos acompanhantes de pacientes de uma clínica-escola de Fonoaudiologia sobre a atuação fonoaudiológica. Métodos: Foram entrevistados 200 sujeitos, 100 do grupo experimental (GE) e 100 do grupo controle (GC). Foi realizada a aplicação de questionário para os acompanhantes dos pacientes (GE) e para indivíduos da população em geral (GC). Os dados foram analisados por meio de frequências absolutas e relativas mostradas em tabelas e gráficos. Para comparação entre GE e GC foi utilizado o teste Qui-quadrado e adotado nível de significância de $5 \%(p<0,05)$. Resultados: Foi observado que tanto o GE quanto o GC apresentaram um conhecimento restrito sobre a Fonoaudiologia, sendo que a imagem do fonoaudiólogo para a população estudada é a de quem trabalha com fala e audição, sendo pouco frequente a menção a outras áreas de atuação. Conclusão: Esperava-se que a população de acompanhantes de pacientes em acompanhamento fonoaudiológico tivesse um maior conhecimento sobre a Fonoaudiologia, pois apresentavam um contato mais amplo com essa profissão; porém, notou-se que, para ambos os grupos, a visão do fonoaudiólogo ainda fica restrita a seu aspecto reabilitador. Assim, os fonoaudiólogos precisam divulgar e orientar mais as pessoas sobre sua profissão.
\end{abstract}

Descritores: Acompanhantes de pacientes; Avaliação; Conhecimento; Área de atuação profissional; Saúde pública

\section{INTRODUÇÃO}

O fonoaudiólogo é um profissional de atuação autônoma e independente, que exerce suas funções nos setores público e privado, é responsável pela promoção da saúde, avaliação, diagnóstico, orientação, terapia (habilitação e reabilitação) e aperfeiçoamento dos aspectos fonoaudiológicos da função auditiva periférica e central, função vestibular, linguagem

Trabalho realizado na Clínica de Fonoaudiologia da Faculdade de Odontologia de Bauru - USP - Bauru (SP), Brasil.

(1) Aprimoranda em Fonoaudiologia em Distúrbios Psiquiátricos da Infância pelo Hospital das Clínicas da Faculdade de Medicina da Universidade de São Paulo - USP - São Paulo (SP), Brasil.

(2) Doutora, Professora do Departamento de Fonoaudiologia da Faculdade de Odontologia de Bauru da Universidade de São Paulo - USP - Bauru (SP), Brasil.

(3) Pós-graduanda (Mestrado) em Fonoaudiologia pela Faculdade de Odontologia de Bauru da Universidade de São Paulo - USP - Bauru (SP), Brasil. Endereço para correspondência: Simone Aparecida Lopes-Herrera. Al. Dr. Octávio Pinheiro Brizola, 9-75, Bauru (SP), Brasil, CEP: 17012-901. E-mail: lopesimone@usp.br

Recebido em: 11/3/2009; Aceito em: 4/8/2009 oral e escrita, voz, fluência, articulação da fala, sistema miofuncional orofacial, cervical e deglutição; podendo também exercer atividades de ensino, pesquisa e administrativa, além de ter atuação clínica, empresarial, escolar (em escola especial e regular), hospitalar, dentre outros ${ }^{(1)}$.

No intuito de compreender o contexto da Fonoaudiologia no Brasil e, particularmente, sua relação com a Educação, em uma breve retomada histórica, pode-se associar o fazer fonoaudiológico a questões políticas, econômicas e educacionais que envolveram seu surgimento e estruturação. A graduação em Fonoaudiologia no Brasil surgiu com o incentivo econômico e político do governo, no modelo educacional da Escola Nova, ligada à atividade pedagógica do professor; já o caráter reabilitador exigiu mais aproximação da área médica. Neste contexto, com a liberdade de se pensar e agir, houve a regulamentação da profissão. Iniciava-se também a realidade mundial do pouco emprego. Assim o surgimento da profissão, a estruturação dos cursos de graduação e a prática do fonoaudiólogo foram e ainda são determinados por necessidades, possibilidades e interesses políticos, econômicos e sociais ${ }^{(2)}$. 
Até pouco tempo atrás, o fonoaudiólogo era visto somente como reabilitador dos distúrbios e não como um promotor de saúde. Mais recentemente, o fonoaudiólogo vem atuando diretamente na promoção da saúde; porém a plena inclusão da Fonoaudiologia nesta área ainda está por vir, apesar do avanço ocorrido $^{(3)}$. Na concepção da prevenção em saúde, o indivíduo é responsabilizado pelo seu estado de saúde ${ }^{(4)}$. Já na visão de promoção em saúde, é suposta uma efetiva participação da população, ao haver uma combinação entre escolhas individuais e responsabilidade social pela saúde, dando ênfase à qualidade de vida ${ }^{(5-7)}$.

Há vários relatos sobre a inserção e o crescimento da atuação do fonoaudiólogo na Saúde Pública no Brasil. Um destes relatos ${ }^{(8)}$ mencionou a existência de legislações que fortalecem as ações relacionadas à Fonoaudiologia no Sistema Único de Saúde (SUS) - como as ações de saúde do trabalhador na atenção básica, ações referentes à promoção de Saúde de forma geral, ações de vigilância e promoção em saúde do trabalhador, instituindo serviços de média e alta complexidade. Apesar de toda a legislação vigente, a inserção do fonoaudiólogo na Saúde Pública requer um profissional comprometido com os princípios de humanização, acolhimento, vínculo e responsabilidade pelo território em que atua e pela comunidade que nele vive. Cabe ao fonoaudiólogo já graduado ampliar sua formação para abraçar essa área de forma responsável e competente.

Outro relato $^{(9)}$ encontrado sobre a atuação fonoaudiológica em Saúde Pública foi de ações realizadas na educação para promoção da saúde através da orientação, em sala de espera, a mães usuárias de unidades básicas de saúde (UBS), sobre o desenvolvimento normal e cuidados gerais com crianças entre zero e dois anos, com ênfase em questões ligadas à amamentação e à remoção de hábitos deletérios ao desenvolvimento infantil. Conclui-se que uma das formas de trabalho da Fonoaudiologia em Saúde Pública é o acolhimento em sala de espera. Esse tipo de atuação contribui para a atuação interdisciplinar, já que em diversas oportunidades contatamse outros profissionais que atenderão o indivíduo, o que pode acrescer debates e questões que passariam despercebidas em consultas clínicas convencionais.

Uma ação que tem se mostrado efetiva na atuação fonoaudiológica na Saúde Pública é o trabalho com agentes comunitários de saúde. Em um dos trabalhos ${ }^{(10)}$ estudados foi proposta a capacitação de agentes comunitários por meio de informações sobre audição e deficiência auditiva, facilitando a identificação destas alterações na população. Os resultados desta proposta comprovaram a eficácia do programa de capacitação com utilização de material e abordagem interativa para os agentes comunitários de saúde dos Programas de Saúde da Família (PSF).

Mesmo havendo toda uma atuação do fonoaudiólogo como profissional que atua em prevenção e promoção de saúde, alguns estudos $^{(11-16)}$ já realizados demonstraram que há um grande desconhecimento por parte de distintas populações com as quais o fonoaudiólogo atua sobre a prática do profissional de Fonoaudiologia.

Estudo de levantamento realizado com profissionais da área da saúde (médicos, psicólogos, fisioterapeutas, terapeutas ocupacionais, dentistas e assistentes sociais), pessoas que frequentam atendimentos fonoaudiológicos em terapia ou na área ambulatorial e seus familiares (todos vinculados a instituições públicas), em que foram questionados sobre os conceitos de saúde geral e saúde fonoaudiológica, mostrou como resultado que, em relação à saúde geral, as dimensões de ordem pessoal prevalecem sobre aquelas meta-pessoais e que o aspecto da comunicação humana exerce pouca influência sobre a saúde geral ${ }^{(11)}$.

Em relação ao desconhecimento que outros profissionais de saúde têm sobre a atuação fonoaudiológica, uma pesquisa realizada com pediatras ${ }^{(12)}$ apontou que eles só têm noção do trabalho dos fonoaudiólogos relacionados aos distúrbios de fala, desconhecendo a atuação fonoaudiológica em outras áreas.

Em outra pesquisa ${ }^{(13)}$ semelhante realizada com médicos e odontólogos, observou-se que foi atribuído à Fonoaudiologia um sentido de área complementar e subordinada a estas áreas. Dessa maneira, esta atribuição leva a acreditar que a Fonoaudiologia, por ser uma área recente de conhecimento, não teve tempo suficiente para se assentar no imaginário dos profissionais médicos e odontólogos, com todas as dimensões que de fato compõem a formação do fonoaudiólogo. $\mathrm{O}$ trabalho em equipe continua sendo um caminho para a integração destes profissionais visando não só um tratamento mais adequado do paciente ${ }^{(14)}$, como também uma maior interação destas áreas de conhecimento.

Um estudo ${ }^{(15)}$ que mostra a importância da atuação em equipe para a divulgação do trabalho do fonoaudiólogo foi um levantamento realizado com ortodontistas e odontopediatras de Belo Horizonte e Itabira; estes profissionais consideraram importante a atuação fonoaudiológica e relataram encaminhar com frequência seus pacientes para tratamento fonoaudiológico. A maioria trabalha com um fonoaudiólogo de confiança e julga importante para adesão ao tratamento atuar no mesmo espaço físico.

A visão restrita sobre a Fonoaudiologia não ocorre somente em relação ao meio médico e odontológico: no ambiente escolar, a visão que se tem da função do fonoaudiólogo ainda é marcada pela sua atividade clínica, mesmo em escolas que possuem fonoaudiólogos, onde a sua função deveria ser bastante clara. Ao fonoaudiólogo só foram atribuídas as funções de tratar e detectar, não sendo reconhecido como um propiciador do desenvolvimento da linguagem ou como alguém que previne o surgimento de alterações da mesma ${ }^{(16)}$. Este fato pode ser elucidado por um estudo ${ }^{(17)}$ que avaliou professores do ensino fundamental a respeito do conhecimento que apresentavam sobre a gagueira. Concluiu-se que eles relacionam o papel do fonoaudiólogo somente à terapia do indivíduo gago, não tendo o conhecimento deste distúrbio da comunicação na questão da prevenção.

Já em relação ao desconhecimento da população em geral sobre a Fonoaudiologia, pode-se citar a realização da Triagem Auditiva Neonatal (TAN), que é garantida por lei, mas pouco aplicada e nem um pouco exigida pela população. A falta de conscientização e informação da população sobre a necessidade e importância da detecção precoce da surdez infantil e a reduzida participação das equipes de saúde, principalmente dos pediatras, na valorização e no encaminhamento para TAN são 
possivelmente alguns dos fatores responsáveis pelo atraso na suspeita e na detecção precoce da deficiência auditiva infantil ${ }^{(18)}$.

Para se conseguir traçar um plano de ação para orientação/ conscientização dos usuários dos serviços de Fonoaudiologia, é preciso conhecer o perfil desta população. No entanto, há poucas pesquisas ${ }^{(19)}$ realizadas com este objetivo. Em uma pesquisa realizada no Ambulatório de Fonoaudiologia do Hospital São Paulo, com intuito de obter o perfil sociodemográfico dos pacientes e acompanhantes e suas percepções sobre a sala de espera e atendimento profissional, foi relatado que a sala de espera era frequentada predominantemente por mulheres, com ensino fundamental incompleto, casadas, com idade média de 39,4 anos e renda familiar de um a três salários mínimos e etnia predominantemente branca. Os frequentadores se mostram satisfeitos com o atendimento recebido, tanto em relação à sala de espera quanto ao atendimento profissional.

Como já relatado, a falta de conhecimento sobre a atuação dos profissionais de saúde não afeta somente a Fonoaudiologia. Em Maringá (PR), foi realizada uma pesquisa com usuários do Serviço Público de Saúde ${ }^{(20)}$, sobre a percepção da relação destes com os profissionais da saúde e revelou-se que os usuários perceberam certo despreparo dos profissionais no atendimento humanitário e, em função disto, destacam-se algumas alternativas que podem contribuir com a melhoria da qualidade dos serviços. Assim, para responder às expectativas dos usuários, o profissional de saúde teria que estabelecer uma comunicação acessível e uma relação mais confiável, levando em consideração o estilo de vida e a história do paciente.

Entra aqui a responsabilidade social do profissional de saúde em não somente realizar seus procedimentos, mas também de explicá-los e descrevê-los aos usuários dos seus serviços. O fonoaudiólogo, como membro de uma equipe, tem formação e conhecimento suficientes para prestar um serviço de acolhimento que atenda às necessidades do usuário, tratando-o como ser humano integral que busca soluções para sua vida em uma relação de ajuda genuína ${ }^{(21)}$.

Tendo por base os estudos anteriormente citados, nota-se que pesquisas que abordem o conhecimento que as pessoas possuem sobre a função do fonoaudiólogo e o acolhimento profissional, sejam elas profissionais da área da saúde ou população leiga, são necessárias para primeiro verificar qual a percepção dos indivíduos sobre qualidade de serviço, se está diretamente ligada ao conhecimento que possuem sobre Fonoaudiologia, e assim, analisar o grau de informação destas pessoas a respeito da atuação fonoaudiológica para, em posse destes dados, propor ações de divulgação que proporcionem visibilidade a esta profissão.

Desta forma, o objetivo desta pesquisa foi avaliar o conhecimento que os acompanhantes de pacientes de uma clínica-escola tinham sobre a Fonoaudiologia e a atuação fonoaudiológica.

\section{MÉTODOS}

\section{Participantes}

Participaram desta pesquisa 200 indivíduos que foram divididos em grupo experimental e grupo controle.
O grupo experimental (GE) foi composto por 100 indivíduos acompanhantes de pacientes que frequentavam a instituição de origem (Clínica-escola de Fonoaudiologia da Faculdade de Odontologia de Bauru). A inclusão destes indivíduos no grupo experimental teve por critério apenas o fato de acompanharem algum paciente da clínica-escola durante atendimento fonoaudiológico (exames audiológicos, avaliações ou terapia fonoaudiológicas).

A maioria dos pacientes atendidos nesta clínica-escola é composta de crianças, adultos e/ou idosos que apresentam problemas neurológicos, sensoriais, de linguagem e/ou aprendizagem e, dessa forma, precisam ser acompanhados até o local de atendimento. Assim, os acompanhantes foram escolhidos para verificar se o fato deles apresentarem um contato maior com a Fonoaudiologia faz com que seu conhecimento sobre essa profissão se diferencie de uma população que não necessariamente apresenta esse contato.

O grupo controle (GC) foi composto por outros 100 indivíduos selecionados aleatoriamente no centro comercial da cidade de Bauru (interior de São Paulo). O centro comercial da cidade foi escolhido por apresentar uma amostra representativa de vários extratos sócio-culturais da cidade na qual foi realizada a pesquisa, conforme orientação estatística sobre seleção de amostras.

\section{Local}

Esta pesquisa foi realizada em dois locais: (1) a coleta de dados do grupo controle foi realizada no centro comercial da cidade de Bauru; (2) para coleta de dados do grupo experimental, o local foi a Clínica-Escola de Fonoaudiologia da Faculdade de Odontologia de Bauru (FOB-USP), da Universidade de São Paulo. A referida clínica-escola atende a uma demanda espontânea de pacientes e também a uma demanda dirigida, que vem encaminhada por outros profissionais ou centros de atendimento (Postos de Saúde, escolas, creches, profissionais de saúde de Bauru e região). As áreas específicas de atendimento fonoaudiológico são estágios supervisionados em diagnóstico fonoaudiológico, que posteriormente encaminham o paciente para os estágios supervisionados em terapias, que se dividem em terapias para distúrbios de linguagem infantil, distúrbio de linguagem em adultos, distúrbio da linguagem escrita, motricidade orofacial, voz e audiologia educacional. Pode também ser realizado o diagnóstico audiológico, que aplica exames específicos (audiometria, imitanciometria e outros) e pode também realizar a indicação e adaptação de AASI (aparelhos de amplificação sonora individual). Após a triagem, o paciente pode também ser encaminhado para outros profissionais, sendo disponibilizados, na referida clínica-escola, atendimentos com psicólogo, neurologista e otorrinolaringologista.

\section{Coleta de dados}

Este estudo foi aprovado pelo Comitê de Ética em Pesquisa da Faculdade de Odontologia de Bauru da Universidade de São Paulo (protocolo n ${ }^{\circ}$ 34/2007). Os participantes tiveram conhecimento do objetivo da pesquisa e dos procedimentos necessários à realização da mesma, concordando com esta $\mathrm{e}$ 
assinando um Termo de Consentimento Livre e Esclarecido (TCLE).

Cada participante respondeu a um questionário composto de seis perguntas, sendo três perguntas abertas e três fechadas. Tais questões foram formuladas para verificar o conhecimento que os entrevistados tinham sobre a Fonoaudiologia e se direcionavam à definição da profissão, áreas de atuação, locais e tipo de trabalho realizado por um fonoaudiólogo, tipos de profissionais com os quais o fonoaudiólogo poderia atuar, quais fases do ciclo vital das pessoas este profissional poderia realizar seu trabalho e qual a importância que este profissional teria na vida do entrevistado e o benefício que este profissional poderia trazer à mesma.

A forma de aplicação dos questionários foi direta, isto é, as pesquisadoras esclareciam os participantes sobre o objetivo da mesma e sobre o TCLE; em seguida, para o grupo experimental, os questionários eram deixados com os indivíduos para serem preenchidos em sala de espera. Para o grupo controle, como a coleta de dados foi no centro da cidade, as pesquisadoras ofereciam aos indivíduos uma prancheta para que os mesmos preenchessem o questionário naquele mesmo instante e ficavam aguardando a finalização do preenchimento.

\section{Análise de dados}

Os resultados foram analisados de forma qualitativa e quantitativa. A análise quantitativa foi realizada por meio de frequências absolutas e relativas. A análise qualitativa dos dados foi feita através da análise individuais das respostas de cada entrevistado, para que se levantassem as categorias emergentes para posterior classificação por categoria de respostas da amostra como um todo. O nível de conhecimento do GE foi avaliado de forma isolada, considerando o papel que eles estavam exercendo junto ao paciente e, posteriormente, foi comparado com o conhecimento do GC. Portanto, para comparar GC e GE foi utilizado, questão a questão, o teste do Qui-quadrado e adotado nível de significância de 5\% (p<0,05 - há diferenças significativas e p>0,05 - não há diferenças significativas). A análise estatística foi aplicada apenas nas categorias que não ultrapassavam 100 respostas, pois algumas questões permitiam que um mesmo indivíduo indicasse mais de uma categoria de resposta. Assim, o valor de p está localizado apenas nas tabelas em que foram realizadas as análises estatísticas, se referindo a probabilidade de significância das variáveis GC e GE.

\section{RESULTADOS}

Nesta exposição de resultado, algumas considerações preliminares devem ser ressaltadas: algumas categorias passaram de 100 respostas, pois o indivíduo poderia indicar mais de uma alternativa. Além disto, como a maioria dos participantes dos grupos experimental (GE) e controle (GC) possuía Ensino Fundamental Completo, os resultados aqui apresentados não variaram em decorrência do nível escolar (Tabela 1).

$\mathrm{Na}$ questão apresentada questionando sobre o que um fonoaudiólogo faz, as respostas dos dois grupos apresentaram algumas diferenças percentuais, ficando evidente que a
Tabela 1. Nível de escolaridade dos participantes

\begin{tabular}{lcc}
\hline Escolaridade & GE & GC \\
\hline Ensino Fundamental completo & $40 \%$ & $31 \%$ \\
Ensino Fundamental incompleto & $18 \%$ & $10 \%$ \\
Ensino Superior completo & $21 \%$ & $30 \%$ \\
Ensino Superior incompleto & $11 \%$ & $23 \%$ \\
Não frequentou escola & $1 \%$ & $0 \%$ \\
Outros & $9 \%$ & $6 \%$ \\
\hline Total & $100 \%$ & $100 \%$ \\
\hline Valor de p & \multicolumn{2}{c}{0,054} \\
\hline
\end{tabular}

Legenda: $\mathrm{GE}=$ grupo experimental; $\mathrm{GC}=$ grupo controle

maioria dos participantes afirmou que a função do fonoaudiólogo é tratar da fala e da audição (Tabela 2), sendo menor a frequência a menção a outras áreas de atuação. Não foram encontradas respostas que indicassem que o fonoaudiólogo tem a função de promover saúde; tendo ficado claro que os papéis de diagnóstico e tratamento (reabilitativo) ainda são os mais predominates na visão da população estudada.

Tabela 2. Atuação fonoaudiológica segundo os participantes

\begin{tabular}{lcc}
\hline Atuação do fonoaudiólogo & GE & GC \\
\hline Fala & $62 \%$ & $65 \%$ \\
Audição & $42 \%$ & $31 \%$ \\
Voz & $21 \%$ & $7 \%$ \\
Não sabe & $15 \%$ & $16 \%$ \\
Motricidade oral & $11 \%$ & $3 \%$ \\
Leitura/Escrita & $9 \%$ & $1 \%$ \\
Linguagem & $8 \%$ & $2 \%$ \\
Comunicação & $3 \%$ & $7 \%$ \\
Deficiências & $1 \%$ & $3 \%$ \\
Várias coisas & $1 \%$ & $0 \%$ \\
Gagueira & $0 \%$ & $2 \%$
\end{tabular}

Legenda: $\mathrm{GE}$ = grupo experimental; $\mathrm{GC}$ = grupo controle

Obs: Não houve análise estatística, dado que a categoria poderia ultrapassar 100 respostas (cada indivíduo poderia indicar mais de uma resposta).

Com relação à questão sobre os profissionais junto aos quais o fonoaudiólogo pode trabalhar, os indivíduos responderam médicos, seguido de psicólogos, dentistas, professores, entre outros. As respostas dadas por ambos os grupos foram semelhantes, conforme mostra a Tabela 3.

$\mathrm{Na}$ questão sobre em quais fases do ciclo vital das pessoas o fonoaudiólogo poderia atuar, comparando as respostas do GE e GC, observou-se que a maioria afirmou que o fonoaudiólogo poderia atuar com indivíduos de todas as idades. Assim, podese definir que o conhecimento de ambos os grupos a respeito desse conteúdo foi semelhante, não sendo estatisticamente significante a diferença (Tabela 4).

De todos os resultados, a questão sobre a importância do papel do fonoaudiólogo na vida de cada participante foi a que mostrou a maior diferença em termos de percentual, pois o GE teve $90 \%$ de respostas afirmativas enquanto o GC teve $41 \%$, conforme demonstra a Figura 1. 
Tabela 3. Profissionais que podem atuar com o fonoaudiólogo

\begin{tabular}{lcc}
\hline Profissionais & GE & GC \\
\hline Médicos & $48 \%$ & $49 \%$ \\
Psicólogos & $40 \%$ & $38 \%$ \\
Não sei & $35 \%$ & $31 \%$ \\
Dentistas & $12 \%$ & $15 \%$ \\
Professores & $10 \%$ & $9 \%$ \\
Fisioterapeutas & $6 \%$ & $8 \%$ \\
Pedagogos & $4 \%$ & $4 \%$ \\
Terapeutas ocupacionais & $3 \%$ & $2 \%$ \\
Nutricionistas & $3 \%$ & $0 \%$ \\
Músicos & $2 \%$ & $3 \%$ \\
Apresentadores & $1 \%$ & $0 \%$ \\
Artistas & $1 \%$ & $1 \%$ \\
Assistentes sociais & $1 \%$ & $3 \%$ \\
Enfermeiros & $0 \%$ & $1 \%$ \\
Jornalistas & $0 \%$ & $1 \%$ \\
Musicoterapeutas & $0 \%$ & $1 \%$ \\
Operadores de telemarketing & $0 \%$ & $1 \%$ \\
\hline
\end{tabular}

Legenda: $\mathrm{GE}=$ grupo experimental; $\mathrm{GC}$ = grupo controle

Obs: Não houve análise estatística, dado que a categoria poderia ultrapassar 100 respostas (cada indivíduo poderia indicar mais de uma resposta).

Tabela 4. Faixa etária dos pacientes com os quais o fonoaudiólogo pode atuar

\begin{tabular}{lcc}
\hline Idades que pode atuar & GE & GC \\
\hline Todas as idades & $89 \%$ & $87 \%$ \\
Todas as idades, menos bebês & $5 \%$ & $6 \%$ \\
Só criança & $2 \%$ & $3 \%$ \\
Crianças e adolescentes & $1 \%$ & $0 \%$ \\
Crianças, adolescentes, jovens e adultos & $1 \%$ & $1 \%$ \\
Só jovens & $1 \%$ & $0 \%$ \\
Jovens e adultos & $0 \%$ & $1 \%$ \\
Todas as idades, menos idosos & $0 \%$ & $1 \%$ \\
Crianças, adolescentes e jovens & $0 \%$ & $1 \%$ \\
Em branco & $1 \%$ & $0 \%$ \\
\hline Total & $100 \%$ & $100 \%$ \\
\hline Valor de p & \multicolumn{2}{c}{0,663} \\
\hline
\end{tabular}

Legenda: $\mathrm{GE}=$ grupo experimental; $\mathrm{GC}=$ grupo controle

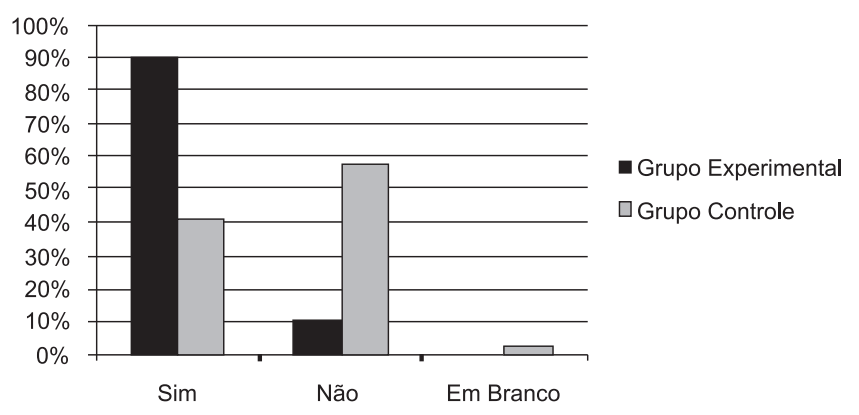

Figura 1. Importância do fonoaudiólogo para o entrevistado
Comparando a questão sobre qual o benefício da atuação do fonoaudiólogo na própria vida, observou-se uma diferença significativa entre os grupos. Para o GE, o maior beneficio apontado foi uma melhor qualidade de vida, acompanhando de uma melhora na comunicação. Já para o GC, o maior benefício se encontrou na melhora nos relacionamentos sociais do indivíduo (Tabela 5).

Tabela 5. Benefícios que um fonoaudiólogo pode trazer para o paciente e sua família

\begin{tabular}{lcc}
\hline Benefícios & GE & GC \\
\hline Qualidade de vida & $27 \%$ & $17 \%$ \\
Melhora da comunicação & $26 \%$ & $11 \%$ \\
Relacionamento social & $21 \%$ & $33 \%$ \\
Bem-estar emocional & $13 \%$ & $2 \%$ \\
Não sei & $13 \%$ & $28 \%$ \\
Saúde bucal & $0 \%$ & $14 \%$ \\
\hline Valor de p & \multicolumn{2}{c}{$<0,001^{*}$} \\
\hline Legenda: GE = grupo experimental; GC = grupo controle \\
*Valor estatisticamente significante
\end{tabular}

\section{DISCUSSÃO}

De forma geral, assim como os pediatras, que acreditavam que o trabalho do fonoaudiólogo estava relacionado aos distúrbios de fala, desconhecendo a atuação fonoaudiológica em outras áreas ${ }^{(12)}$, grande parte dos entrevistados desta pesquisa apresentou um conhecimento sobre Fonoaudiologia restrito à fala e audição, apesar de existir atuação do fonoaudiólogo em outras áreas, como a linguagem, motricidade oral, voz e saúde coletiva. Este resultado confirma a afirmação feita pela literatura ${ }^{(3)}$ que o fonoaudiólogo ainda é visto somente como um reabilitador dos distúrbios e não um promotor de saúde, mesmo pela própria população que ele atende.

Como já citado, o fonoaudiólogo é responsável pela promoção da saúde, avaliação, diagnóstico, orientação, terapia (habilitação e reabilitação) e aperfeiçoamento dos aspectos fonoaudiológicos ${ }^{(1)}$. Ao analisar os questionários, não foram encontradas respostas que indicassem que o fonoaudiólogo tem a função de promover saúde, apenas de diagnosticar e tratar. A população analisada atribuiu somente as funções de tratar e detectar, não sendo o fonoaudiólogo reconhecido como um propiciador do desenvolvimento da linguagem e da comunicação ou como um profissional que previne o surgimento das alterações das mesmas ${ }^{(16)}$.

Neste trabalho, em relação às áreas de atuação do fonoaudiólogo, embora tenham sido encontradas respostas que se referiam a praticamente todas as áreas em que este profissional pode atuar (como audição, linguagem oral e escrita, voz, fluência, articulação da fala, sistema miofuncional orofacial, entre outras), a maioria dos entrevistados mostrou um conhecimento restrito sobre essa profissão, indicando apenas a fala e a audição. Isso pode ter ocorrido pelo fato citado na literatura ${ }^{(13)}$ onde alguns profissionais definiram a Fonoaudiologia como uma área complementar e subordinada às áreas médica e odontológica. 
A relação dos fonoaudiólogos com outros profissionais foi citada, mas mesmo esta pesquisa tendo sido realizada em uma clínica-escola de Fonoaudiologia que se encontra dentro de uma Faculdade de Odontologia, os entrevistados não demonstraram possuir conhecimento da relação entre estas duas profissões, pois poucos (12\% GE e $15 \% \mathrm{GC})$ responderam que os dentistas podem trabalhar junto ao fonoaudiólogo. A maioria acredita que a parceria do fonoaudiólogo ocorre somente com médicos e psicólogos.

Os indivíduos do GE relataram que reconhecem a importância da Fonoaudiologia em sua vida, o que era esperado porque, como acompanhantes de pacientes que realizam atendimentos fonoaudiológicos, estes indivíduos apresentam um contato maior com a Fonoaudiologia. Já o GC apresentou um número menor de indivíduos que reconheceram a importância dessa profissão. Portanto, caberia à classe dos fonoaudiólogos divulgar de forma mais ampla para a população a importância da atuação fonoaudiológica, utilizando sempre linguagem simples e acessível, explicando o que é a profissão, quais as áreas de atuação e quais possibilidades de promoção e prevenção dentro da Fonoaudiologia.

Da mesma maneira, os acompanhantes apresentaram um conhecimento restrito sobre Fonoaudiologia, o que pode significar um despreparo por parte dos fonoaudiólogos em relação à orientação, tanto dos pacientes quanto de seus acompanhantes, o que demonstra uma comunicação pouco eficiente ou restrita com os mesmos. Para modificar este quadro, o fonoaudiólogo precisa sempre estar em contato com a família dos indivíduos com os quais atua, explicando seu trabalho, apresentando os benefícios para a qualidade de vida, além de se mostrar acessível sempre para que o paciente e seus acompanhantes tenham liberdade para tirar suas dúvidas. É importante que todo fonoaudiólogo, ao iniciar um tratamento, explique sobre sua profissão e sua área de atuação e esclareça o paciente e seus familiares sobre todo e qualquer procedimento realizado, aproveitando o espaço possível de atuação para proporcionar, não só o conhecimento sobre sua própria atuação, mas também de promoção e prevenção em saúde de uma forma geral.

\section{CONCLUSÃO}

Para ambos os grupos (experimental e controle), a imagem do fonoaudiólogo é a de quem trabalha, principalmente, com fala e audição, visando melhorar a comunicação, a qualidade de vida e o relacionamento social do indivíduo. Embora esta visão esteja correta, ainda é restrita em relação a tudo o que um fonoaudiólogo pode realizar dentro de sua profissão e o que pode possibilitar à população em termos de promoção e prevenção de saúde.

Assim, os fonoaudiólogos precisam divulgar e orientar mais sobre sua profissão, utilizando - como classe profissional - os meios de comunicação para atingir toda população e, de forma mais direta, esclarecer seus atendimentos aos pacientes e seus respectivos acompanhantes por meio de uma comunicação acessível (folhetos informativos, painéis, palestras de dinâmicas de sala de espera, etc.) a todas as classes socioeconômicas. Uma forma de divulgação e orientação interessante de ser realizada é aproveitar datas específicas da Fonoaudiologia (Dia da Voz, Dia da Gagueira, Dia do Fonoaudiólogo, Dia Internacional de Conscientização do Ruído, Dia da Dislexia, entre outros) para promover campanhas nacionais divulgando estas áreas, o trabalho de sua área profissional, além de executar ações de promoção e prevenção de Saúde em todo e qualquer local em que atue, aproveitando os espaços (que não só o terapêutico) sempre que possível.

Desta forma, o conhecimento da população sobre Fonoaudiologia poderá ser ampliado e, consequentemente, a sua valorização como profissão de saúde que atua na qualidade de vida das pessoas e na promoção da saúde.

\section{AGRADECIMENTOS}

À Fundação de Amparo à Pesquisa do Estado de São Paulo (FAPESP) pelo apoio financeiro concedido por meio de projeto de iniciação científica, e à Universidade de São Paulo (USP) e à Faculdade de Odontologia de Bauru (FOB-USP) pela oportunidade de realizar essa pesquisa.

\begin{abstract}
Purpose: To assess the knowledge that persons accompanying patients in a Speech-Language Pathology school-clinic have about the work of speech-language pathologists. Methods: Two hundred subjects were interviewed, 100 from the experimental group (EG) and 100 from the control group (CG). A questionnaires was handed to persons accompanying patients (EG) and to random individuals (CG). Data were analyzed using absolute and relative frequencies shown in tables and figures. In order to compare EG and CG, the Chi-square test was used, with a significance level of $5 \%(\mathrm{p}<0.05)$. Results: Both the experimental and the control groups revealed restricted knowledge regarding Speech-Language Pathology. The speech-language pathologist's image, to the studied population, is that of a person who treats speech and hearing; mention to other areas of work was infrequent. Conclusion: It was expected that persons accompanying patients during Speech-Language Pathology treatment would have greater knowledge about Speech-Language Pathology than general population, because they have had a wider contact with this profession. However, it was observed that, for both groups, the image of the speech-language pathologist was restricted to its rehabilitative aspect. Therefore, speech-language pathologists need to further promote and instruct those around them about their profession.
\end{abstract}

Keywords: Patient escort service; Evaluation; Knowledge; Professional practice location; Public health 


\section{REFERÊNCIAS}

1. Conselho Federal de Fonoaudiologia. Exercício profissional do fonoaudiólogo [Internet] 2002 [citado 2010 Jan 10]. Brasília (DF): CFF; 2002. Disponível em: http://www.crfa8r.org.br/downloads/epdo1.pdf

2. Bacha SM, Osório AM. Fonoaudiologia e educação: uma revisão da prática histórica. Rev CEFAC. 2004;6(2):215-21.

3. Lipay MS, Almeida EC. A fonoaudiologia e sua inserção na saúde pública. Rev Ciênc Méd. 2007;6(1):31-41.

4. Buss PM. Uma introdução ao conceito de promoção da Saúde. In: Czeresnia D, Freitas CM. Promoção da saúde: conceitos, reflexões, tendências. Rio de Janeiro: Fiocruz; 2005. p.15-38.

5. Penteado RZ, Servilha EA. Fonoaudiologia em saúde pública/coletiva: compreendendo prevenção e o paradigma da promoção da saúde. Distúrb Comun. 2004;16(1):107-16.

6. Alves VS. Um modelo de educação em saúde para o Programa Saúde da Família: pela integralidade da atenção e reorientação do modelo assistencial. Interface (Botucatu). 2005;9(16):39-52.

7. Oliveira DL. A 'nova' saúde pública e a promoção da saúde via educação: entre a tradição e a inovação.Rev Latinoam Enferm. 2005;13(3):423-31.

8. Azevedo BA. Fonoaudiologia na saúde coletiva: uma área em crescimento [Editorial II]. Rev CEFAC. 2007;9(2).

9. Lenz AJ, Gernhardt A, Goulart BN, Zimmer F, Rocha JG, Vilanova JR et al. Acolhimento, humanização e fonoaudiologia: relato de experiência em unidade básica de saúde de Novo Hamburgo (RS). Boletim da Saúde [Internet] 2006 [citado 2010 Fev 5];20(2). Disponível em: http://www. esp.rs.gov.br/img2/v20n2_09Acolhimento.pdf

10. Alvarenga KF, Bevilacqua MC, Martinez MA, Melo TM, Blasca WQ, Taga MF. Proposta para capacitação de agentes comunitários de saúde em saúde auditiva. Pró-Fono. 2008;20(3):171-6.

11. Andrade CR. Conceito de saúde e saúde fonoaudiológica: uma análise dos discursos dos profissionais de saúde e dos usuários do serviço de Fonoaudiologia. [dissertação]. São Paulo: Universidade de São Paulo, Faculdade de Filosofia, Letras e Ciências Humanas; 1991.
12. Rabelo BG, Salomão LM, Carnivali PA, Leite IC. Algumas considerações sobre o grau de conhecimento dos pediatras sobre questões fonoaudiológicas. Fono Atual. 2004;27(7):4-10.

13. Calais LL. Conhecimento dos professores do ensino fundamental sobre a gagueira. Pró-Fono. 2002;14(1):23-30.

14. Silveira PC, Cunha DA, Fontes ML, Lima AE, Farias OS, Lucena JA. A importância da prevenção à gagueira nas escolas. Fono Atual. 2002;22(5):12-27.

15. Moreira DR, Friedman S. Fonoaudiologia: o sentido que se produz nas áreas médica, odontológica e fonoaudiológica. Pró-Fono. 2002;14(1):129-36.

16. Amaral EC, Amaral EC, Bacha SM, Ghersel EL, Rodrigues PM. Interrelação entre a odontologia e a fonoaudiologia na motricidade orofacial. Rev CEFAC. 2006;8(3):337-51.

17. Varanda CP, Campos LG, Motta AR. Adesão ao tratamento fonoaudiológico segundo a visão de ortodontistas e odontopediatras. Rev Soc Bras Fonoaudiol. 2008;13(3):233-9.

18. Gatto CI, Tochetto TM. Deficiência auditiva infantil: implicações e soluções. Rev CEFAC. 2007;9(1):110-15.

19. Andrade A, Marteleto MR, Pedromônico MR. Perfil sociodemográfico dos usuários do ambulatório de fonoaudiologia. Fono Atual. 2005;33(8):34-40.

20. Marcon SS, Soares NT, Sassá AH. Percepção dos usuários sobre suas relações com os profissionais de saúde. Online Braz. J. Nurs [Internet] 2007 [citado 2010 Fev 2];6(3). Disponível em: http://www.uff.br/ objnursing/index.php/nursing/article/view/j.1676-4285.2007.996/260

21. Almeida EC, Furtado LM. Acolhimento em saúde pública: a contribuição do fonoaudiólogo. Rev Ciênc Méd. 2006;15(3):249-56. 